Journal of Advanced Research in Fluid Mechanics and Thermal Sciences

Journal homepage: www.akademiabaru.com/arfmts.html ISSN: 2289-7879

\title{
Transient-Based Leak Detection and Monitoring of Water Pipes Using Complementary Ensemble Empirical Mode Decomposition (CEEMD) Method
}

\author{
Muhammad Aminuddin PiRemli ${ }^{1,2}$, Mohd Fairusham Ghazali ${ }^{1,2,3,}{ }^{*}$, W. H. Azmi ${ }^{1,4}$, M. Y. Hanafi ${ }^{1,2}$ \\ Faculty of Mechanical and Automotive Engineering Technology, University Malaysia Pahang, 26600 Pekan, Pahang, Malaysia \\ Advance Structural Integrity and Vibration Research (ASIVR), University Malaysia Pahang, 26600 Pekan, Pahang, Malaysia \\ 3 Centre of Excellence for Advanced Research in Fluid Flow (CARIFF), University Malaysia Pahang Lebuhraya Tun Razak 26300 Gambang Kuantan, \\ Pahang, Malaysia \\ 4 Automotive Engineering Centre, University Malaysia Pahang, 26600 Pekan, Pahang, Malaysia
}

\section{ARTICLE INFO ABSTRACT}

\section{Article history:}

Received 30 December 2020

Received in revised form 26 April 2021

Accepted 4 May 2021

Available online 17 June 2021

\begin{abstract}
Transient signal occurs when there is sudden change of pressure inside the pipeline especially due to pressure surge or opening and closing valve. Decomposition method applied in this study to analyze the transient signal and remove noise that contaminate the signal. In this paper, an adaptive decomposition algorithm that is Complementary Ensemble Empirical Mode Decomposition (CEEMD) proposed in order to overcome the problem occur in Hilbert-Huang Transform (HHT) method. This method takes over the Empirical Mode Decomposition (EMD) method that purpose as pre-processing method in HHT. This improvement made to overcome mode mixing and reconstruction error that lies in EMD method. CEEMD method used to extract the IMF's component of a contaminated signal to remove undesirable noise signal. Instantaneous analysis using Hilbert Transform (HT) apply for selected IMF to locate the feature's position along the pipeline. The result prove that proposed decomposition method show success with the percentage of error between measured and analysed distance only below that $4 \%$ for all features extracted.
\end{abstract}

Keywords:

Leak detection; signal processing; decomposition; CEEMD

\section{Introduction}

Pipeline water distribution system is very important because the quality of life of humans has been increased by transporting fluid from one point to another. For instance, the use of the piping system as a fluid carrier from damp to every house also made it easier for the industrial user to transport fluid such as water. Pipe leakage is a major operational problem in the world. Due to poor quality and defective pipe materials, pipe breaks resulting from poor workmanship, operational errors such as excessive pressure, quick closing or opening valves, corrosion, leaking fittings, and accidental or intentional damage to fixtures, leaks may occur [1]. Leakage from external pipes and by

\footnotetext{
* Corresponding author

Email address: fairusham@ump.edu.my
}

https://doi.org/10.37934/arfmts.83.2.135148 
visual inspection and the use of certain devices is easily identified. The underground pipes are however another challenge to track the leakage, so the small in-line sensor should be useful [2].

There should be losses due to pipeline inadequacies which are physical losses such as leakage and apparent losses like water thieves and measurement inaccuracy [3]. The detection, location, and remediation of these leaks is a difficult task since most problems receive little or no customer attention, and there is often no leakage management program until an emergency is present or when a deficiency arises. The need for leak detection tools was realized early in the 19th century when manual sounding based on wooden listening sticks was first in use. This was followed by more advanced technologies as described by the International Water Association (IWA) Water Loss Group in this leakage detection technology timeline, with a technological boost in the 2000s which included the penetration of acoustic loggers, digital correlators, and advanced ground microphones. There is no doubt that these developments made leak location efforts more effective and still help reducing water loss rates at that time. From day to day many new techniques have been proposed [4].

The timeframe for leak detection technology is shown in Figure 1 below. Based on Figure 1, the method focused more on sound techniques in the 1850s. This method takes a long time to detect the leak. By the 1900s, more electrical devices were used to detect leaks. The equipment and technology for the detection of leakage were developed until 2003 when advanced ground microphones were developed. Conventional ground penetrating radar, it based on three concepts, which are tomography, wave amplitude reflection and electromagnetic wave intensity, are applied [5]. In 2008, new technology introduces by placing a smart ball inside the pipeline system and travel along the system to create a mapping of pipeline structure and discover the intermittent contain in the pipeline. The issue of this technology, its need expert user in operational and also high in cost. Some researcher also focused on acoustic-based signal method. Some of the acoustic devices are based on the fact that noise is created due to turbulence when a leak occurs, its sensors listen to the different noise signals and cause an alarm [6-8].

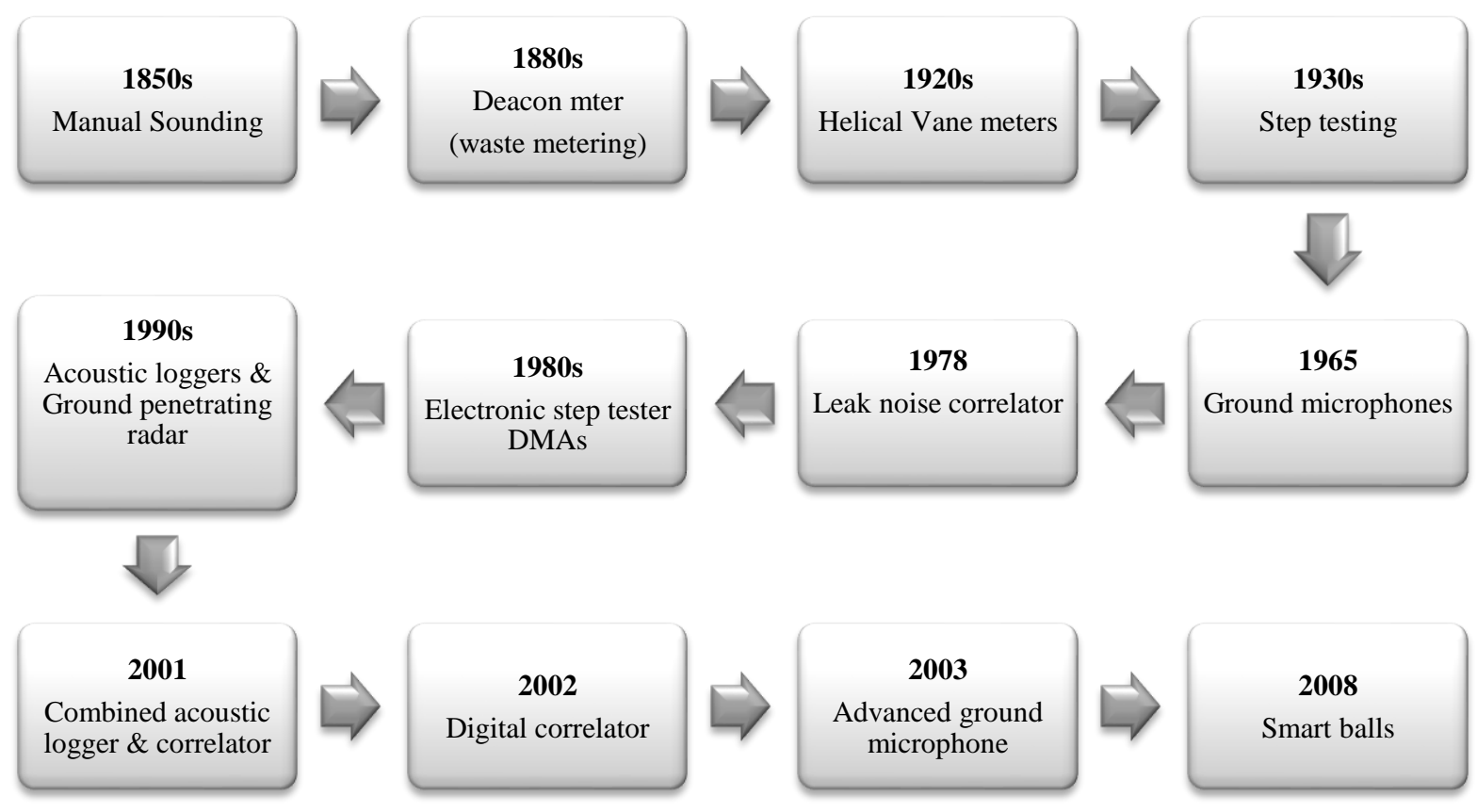

Fig. 1. Chronology of leak detection technology 
Researchers have now switched to various leak technology levels to minimize cost and time in detecting and repairing pipe failure, in particular for the underground pipeline system. The pressure transient approach is one of the world's most popular methods among researchers since the transient wave in the pipeline is the main interest. Transient wave-based methods were used to send active waves, collect the data from field instruments, and mathematically or theoretically analyze information to estimate vulnerabilities in the pipeline system [9-13]. Transient waves can appear inside the pipe by a sudden change of water pressure either by opening or closing the valve or from other sources that can disturb the flow of water inside the pipeline. This approach can be said to be ecofriendly and also low cost and most important the detection of the leak location is also accurate. But researchers in this area are searching for the best way to interpret the data collected during the process. In general, data collection during the process is contaminated by other factors, such as the vibration of the pipe, pump interference, and external noise. True pipe structures have various unknown parameters, limited data sets, and very high noise [14]. This provides an interface with the investigator to establish a system for signal processing, which can extract all contaminated signal and reconstruct the true wave signal captured by the sensor.

\section{Signal Processing}

Signal processing is the study and modification of a signal to maximize or enhance its quality or efficiency. To generate a signal of better quality than the original signal, numerous mathematical and computational algorithms are employed to create a useful signal. Leakage in the pipelines is becoming a major issue. Rapid and intelligent steps must be taken to mitigate loss and the consequences of leakage. A conventional approach such as visual inspection can be used, however, it takes a longer time to process. It also lacks reliability and can lead to erroneous detection of the leak. The signal analysis approach is being used to find and locate the leak location. Therefore, the leaks can be fixed in no time. Signal analysis results are a technique by which data from the pipe system can be analyzed and acquired by the sensor mounted on the pipeline. In the data processing system, normalization, transformation, selection, and extraction features were included [15]. This paper used Hilbert-Huang Transform (HHT) as a method to analyze the signal and obtain the location of features along with the pipeline network

\subsection{Hilbert-Huang Transform (HHT)}

In the combination of empirical mode decomposition (EMD) and the Hilbert spectral analysis (HSA), descriptions of the Hilbert-Huang transform (HHT) are indicated in [16-18]. It is an adaptive approach for data analysis designed primarily for the interpretation of non-linear and non-stationary data. The main part of the HHT is the EMD method which can decompose any complicated data set into some number of intrinsic modes (IMF). This method of decomposition in the time domain is adaptive and thus highly efficient.

As the decomposition is based upon the local time scale of the data, it suitable to applies to nonlinear and non-stationary processes. With the transformation of Hilbert, the IMF produces instantaneous frequencies as time functions that give clear identification of integrated structures. The EMD produces time series components, which transformations using Hilbert that lead to meaningful definitions of these two instantaneous and explanation of the time series by the combination of HT and EMD [19]. However, in this paper, the new approach is shown by replacing EMD in HHT by CEEMD. CEEMD is the next generation of EMD that can overcome the mode mixing 
problem that usually occurs in the decomposition process. This will create a new HHT version with a combination of CEEMD and HT.

\subsection{Complementary Ensemble Empirical Mode Decomposition (CEEMD)}

The development of CEEMD by Yeh et al., [20] is mainly focused on improving decomposition methods of EMD and EEMD. Although EEMD is improving dramatically and can greatly improve stability compared with EMD, it cannot fully neutralize additional noise. CEEMD ensures the decomposition process as well as EEMD and can reduce the reconstruction error caused by additional white noise by pairing noise containing positive and negative signals [20]. CEEMD is an enhanced version with a more robust design to overcome "mode mix". At each stage of the decomposition, a particular white noise is assigned in CEEMD [21]. The following is the CEEMD algorithm

(i) Add white noise $W^{j}(n)(j=1,2, \cdots T)$ into time series

$X^{j}(n)=X(n)+w^{j}(n)$

(ii) Decompose $X(n)(j=1,2, \cdots T)$ and compute

$\overline{\operatorname{Im} f_{1}}(n)=\frac{1}{T} \sum_{j=1}^{T} i m f_{1}^{j}(n)$

(iii) Calculate residue

$r_{1}(n)=x(n)-\overline{\operatorname{Im} f_{1}}(n)$

(iv) Decompose, $r_{1}(n)=x(n)+\lambda_{1} E_{1}\left(w^{j}(n)\right)(j=1,2, \cdots T)$ and compute

$\overline{\operatorname{Im} f_{2}}(n)=\frac{1}{T} \sum_{j=1}^{T} E_{1}\left(r_{1}(n)+\lambda_{I} E_{1}\left(w^{j}(n)\right)\right)$

(v) For $s=2,3, \ldots, \mathrm{S}$, compute the $s^{\text {th }}$ residual

$r_{s}(n)=r_{s-1}(n)-\overline{\operatorname{Im} f_{s}}(n)$

(vi) Decompose, $r_{s}(n)+\lambda_{s} E_{s}\left(w^{j}(n)\right)(j=1,2, \cdots T)$ and compute

$\overline{\operatorname{Im} f_{s+1}}(n)=\frac{1}{T} \sum_{j=1}^{T} E_{1}\left(r_{s}(n)+\lambda_{s} E_{s}\left(w^{j}(n)\right)\right)$

(vii) Iterate step (v.) and (vi.) for next s. Observe that the $\lambda_{s}(s=1,2, \ldots \mathrm{T})$ can be used to select

proper SNR at each step. 
CEEMD solves the problem of mixing mode thoroughly and reduces iterations better. By introducing adding adaptive noise inside the signal and also performing the averaging calculation process, this method diminishes the mode mixing problem that occurs in the previous generation of CEEMD [22]. Theoretical analysis and experiments show that CEEMD offers a precise reconstruction of time series and better spectral separation of IMFs than the other two methods of decomposition in the empirical mode. Every IMF component represents signal characteristics in various bands of high to low frequencies, and the IMF's contain all frequency components [23].

\section{Methodology}

The key approach in this analysis would be the transient method. This approach is essentially one of the methods widely used in the method of leak detection. It is the concept of using transient pressure flow. Pressure transient occurred when the flow abruptly shifted when the valve in the pipelines was closed or opened. This triggers wave propagation in the pipelines and is used as a way of detecting leaks. The latest transient approach technique is by using two sensors positioned opposite between the leaks. The downside of this approach is that the two sensors operate the necessary task. Besides, both sensors should also hear the noise to detect leakage, so that the distance between the sensors is important to detect the leak successfully. Uncertainty such as a leak can cause a sudden pressure change or drop in a positive pressure surge. Signal and leak-reflected signals could take several paths due to boundary echoes in a transient wave that travel along with the pipeline network [24].

To overcome the problem from the present technique, this study has proposed a new method or techniques based on the present transient method. The equipment has been modified by using just only one sensor which is a pressure sensor place in a fire hydrant cap as the point of analysis. Pressure sensor is function to capture the infromtation from the reflected wave that trigger by using solenoid valve which also attached to the fire hydrant cap. Piezoelectric pressure sensor got a high frequency that has the capabilities to measure the response of ruggedness, pressure change, extended range, high stiffness, and can measure quasi-static pressures [25]. The idea is that as the pressure transient passes through the pipe and the leaks are contacted, the signal is echoed. The pressure sensor collects this signal. The pressure sensor is a device that converts analog input such as strain, stress, or pressure of a certain material into an electrical signal which is then converted into a digital input by computer software. Then, the data will be analyzed by using a method of signal processing. The benefits of this proposed method are that the accuracy of detecting leaks improved. Most importantly, this type of method is a friendly user because all of the equipment is in the same place. So, the number of operators can be reduced and also does not need an expertise to handle.

\subsection{Pipeline System Setup}

The pipeline setup for the experiment includes the water tank, pump, solenoid valve, piezoelectric pressure sensor, and MDPE pipe to create a pipeline system of $152.30 \mathrm{~m}$ long. The Medium Density Polyethylene (MDPE) pipe used in the experiment with an external diameter of the pipe is $60 \mathrm{~mm}$ and the internal diameter is $55 \mathrm{~mm}$. The hole drilled at a certain distance to be assumed as an artificial leak in the pipeline network. The artificial leak cover inside a box to reduce the noise that can disturb the signal capture by the pressure sensor. The distance of the fire hydrant (point of analysis) to the leak and outlet was $47.87 \mathrm{~m}$ and $93.40 \mathrm{~m}$ respectively. The overview of the pipeline system setup is shown in Figure 2. The water tank and pump was placed far from fire hydrant to avoid noise contaminated the signal captured. Solenoid valve attached to a fire hydrant as a function to 
generate "water hammer" phenomenon to robust with a real live water distribution system that produces pressure surge during the operating system.

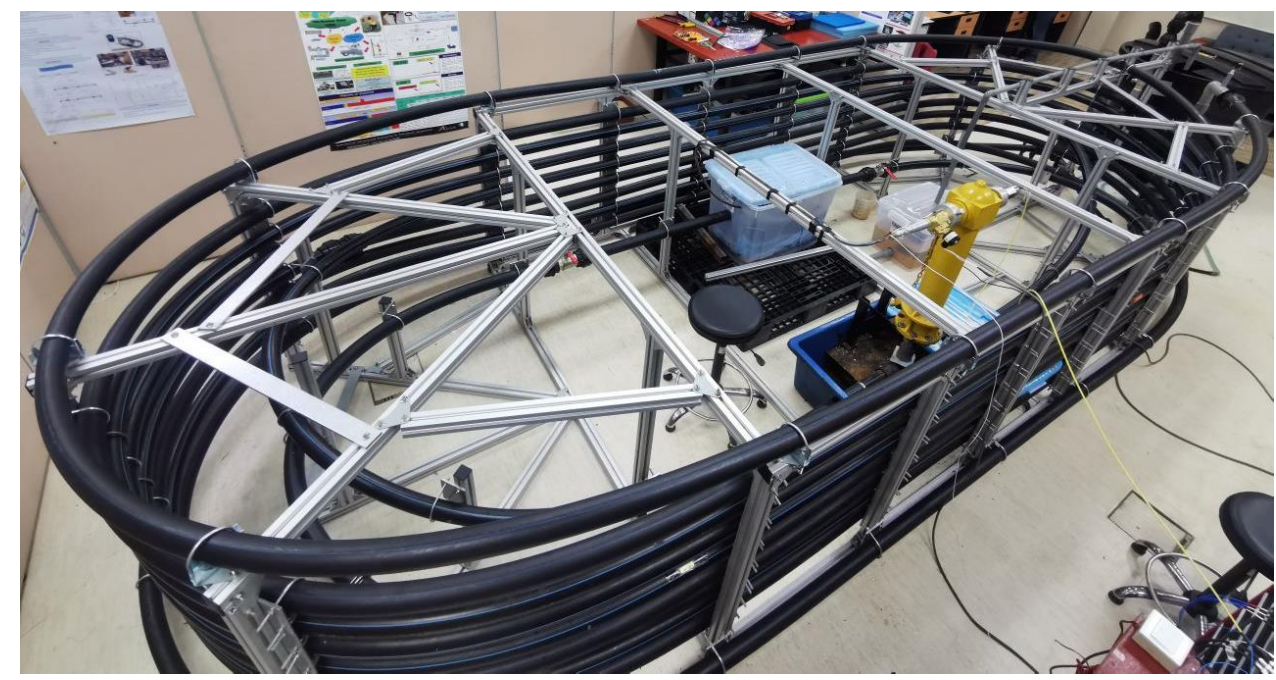

Fig. 2. Lab-scale setup of the pipeline system

Water hammer creates pressure surge and the wave travel along the pipeline system. The transient signal that travel will create a reflection when the wave passes through the features and also a blockage inside the pipeline. The design of the fire hydrant cap with attached solenoid valve and pressure sensor is shown in Figure 3(a) and Figure 3(b). The point of an analysis is designed to be a little bit far from the pump to reduce the noise generate by the pump contaminate the signal and to avoid the turbulent flow of water produce by a high-pressure pump [26].

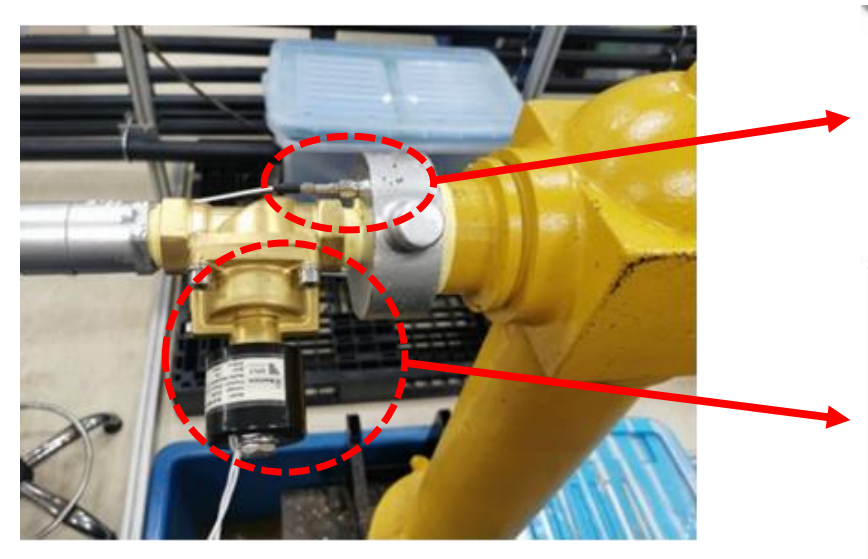

(a)

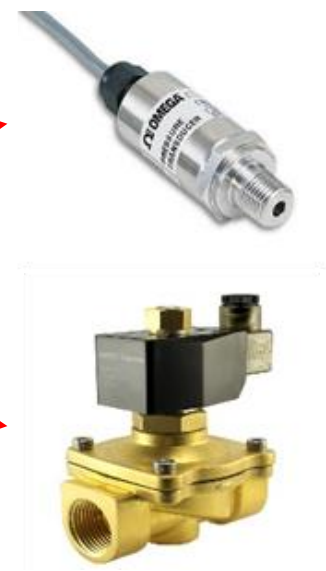

(b)

Fig. 3. (a) Point of analysis, (b) Pressure sensor and solenoid valve attached to fire hydrant cap

Once the pump was on, the water will let to be flow and pressurized inside the pipe to maintain the water pressure inside the pipe. Once the pressure achieve, the solenoid valve will opened and closed in a millisecond interval to generate water hammber and pressure signal. This signal will travel along the pipeline network and will create a reflection signal when it pass through any features. These reflection signal will be captured by pressure sensor as and information of the pipeline. These raw signal then converted into the DASYLab directly. From here, the data will be transfer into Matlab sand will undergo signal processing analysis which are CEEMD as pre-processing and HT as postprocessing. 


\subsection{Signal Analysis}

The data collected basically will include everything from inside and outside the pipeline. The noise from the surrounding environment, pump, pipe vibration, pipe friction, and others will contaminants the original signal collected thru the pressure sensor. At this stage, the signal processing method is the crucial part to remove the unwanted signal and analyze the data for features extraction. The flow chart of the data collection and data analysis is shown in Figure 4. The signal captured directly from pressure sensor will be converted into Matlab software. The data will be decomposed using CEEMD method generally by using coding in the Matlab. Decompostion will generate several Instrinsic Mode Function (IMF) component. The number of component depending on the data itself. If the amplitude is high the component will increase. However, from all the component, only selected IMF will be used to undergo Instantaneous Frequency Analysis (IFA) by using Hilbert Transfrom (HT). From here we can localised the location of feature such as leak and outlet.

\subsubsection{Instantaneous frequency analysis using Hilbert Transform (HT)}

As a signal processing concept, the instantaneous frequency (IF) was originally defined in the framework of FM modulation theory in communications. Many researchers have contributed in this area to focused on instantaneous frequency (IF) such as Ackroyd [27], Griffiths [28], Gupta [29], Jones and Boashash [30], and Rihaczek [31]. Rihaczek [31] studied the opinions in the time-frequency domain of his complex energy distribution where the signal is focused. In the research, the researcher proved that the energy was focused in frequency about the IF. Instantaneous frequency analysis used in this paper Hilbert transform (HT). HT will analyze the signal of selected IMF after the decomposition process using CEEMD. The peak or spike that present clearly in the result shows that there are features or uncertainty at that point inside the pipeline network. However, the result will be analyzed in the time interval. The time interval where the spike appears is then converted to the distance using Eq. (7) with the speed of sound (SOS) $524 \mathrm{~m} / \mathrm{s}$. Then the distance was compared between the measured distance from the test rig and the analyzed distance from the analysis. The equation to convert time into the distance as follow

Distance $(m)=[($ Time,$t(s)) \times(S O S)] \div 2$ 


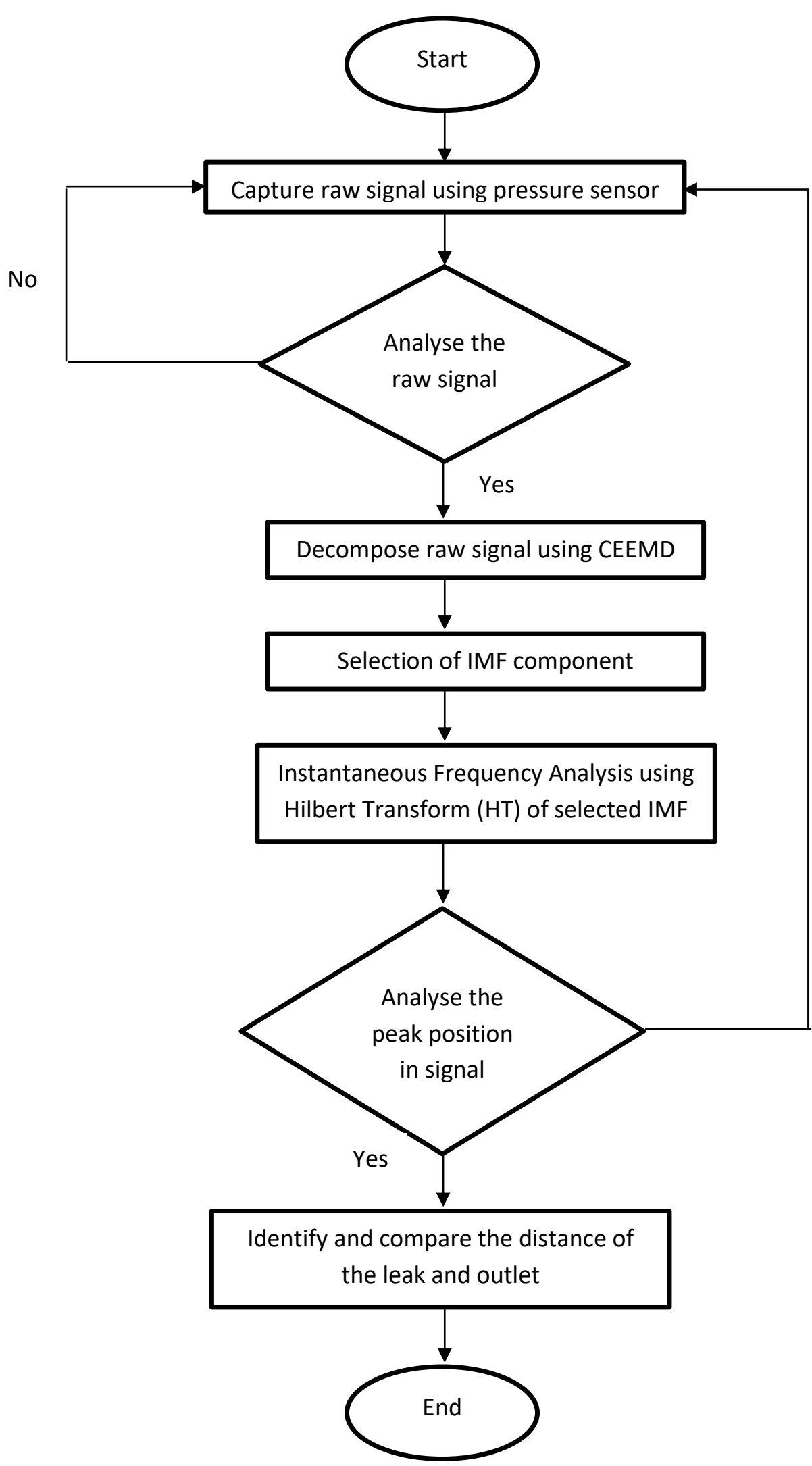

Fig. 4. Flow chart of data collection and analysis 


\section{Result and Discussion}

In essence, the raw data provided no information on the pipelines due to the high noise level, as shown in Figure 5. The signal was captured for 30000 samples with a sampling frequency of 10000. The data recorded for 3 second of time to ensure that the signal captured does not miss any information of the pipeline system. The signal showed a spike at the 10000 samples which represents the point when the solenoid valve opened.

Thus, the pressure inside the pipe drops gradually until the solenoid valve closed. At this point, the pressure begins to raised again until it reached the level of pressure manipulated inside the pipeline. The pressure drop will increase with the increase of pressure inside the pipeline. The pressure inside the pipe was controlled and manipulated by only using the valve near the oulet. The pressure was monitor at two point along the pipeline that were near the water pump directly after the tank and the other one is before the oulet near the valve control point.

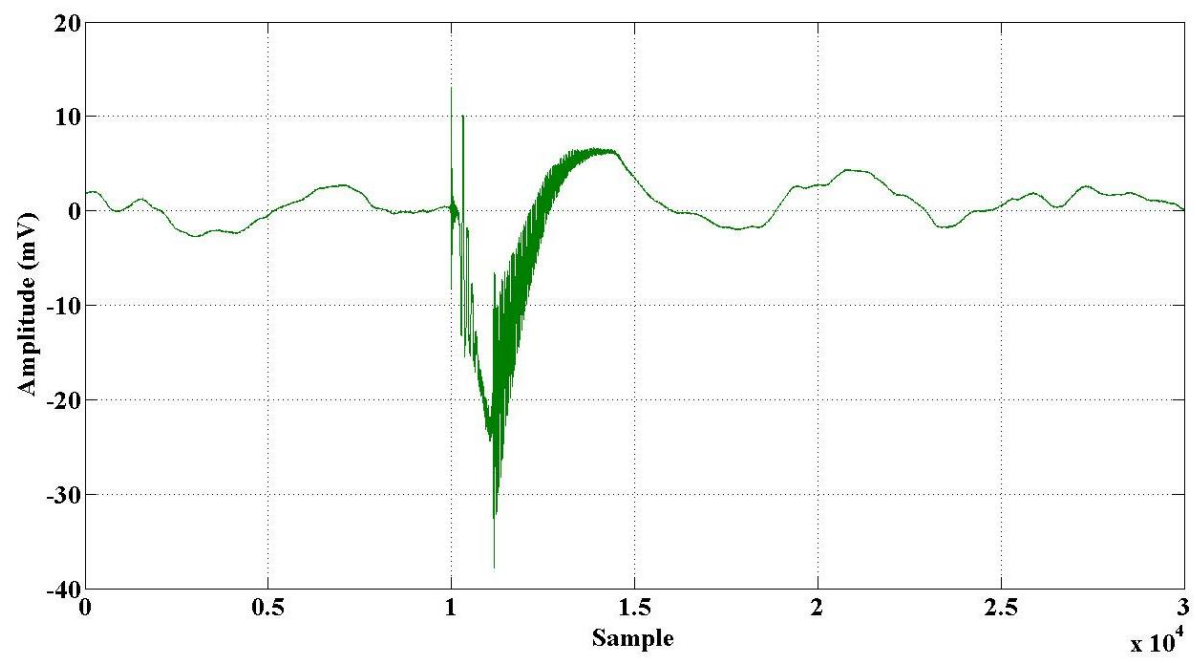

Fig. 5. Raw data captured by the pressure sensor

The raw signal is contaminated with all types of noise such as pump noise, pipe vibration noise, surrounding noise, and others. The true signal is hidden inside the signal that contains noise. Thus, signal processing is used in this article to remove all unwanted signals captured by the pressure sensor.

\subsection{Decomposition Method Using CEEMD}

The decomposition method plays an important part to overcome the problem of the contaminated signal captured during the process. By using the decomposition method of CEEMD, the data obtained by using a pressure sensor decomposed into several components of IMFs to eliminate the undesirable signal within the raw data. CEEMD method decomposes the data to provide a better view of the signal inside the raw data and to be selected for instantaneous frequency analysis as shown in Figure 6. IMFs of levels 1, 2, and 3 contain noise with high amplitude. Meanwhile, IMFs 8 and above usually contain the basic network response and also the residue. So, all this level of IMFs will be removed for further analysis. IMFs level 5 until level 7 considered as the best level of IMF to be selected and use for IF analysis [32]. 
Intrinsic Mode Function
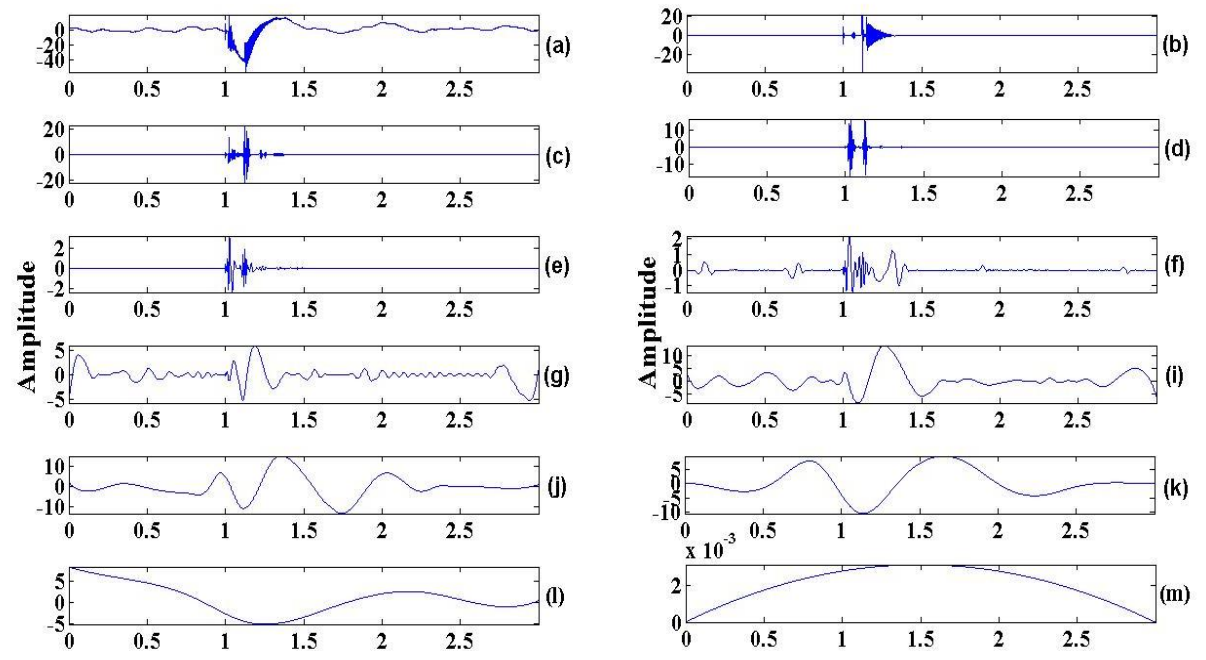

Fig. 6. IMFs component using CEEMD method

\subsection{Instantaneous Frequency Estimation of 1 bar Signal}

Figure 7(a) and Figure 7(b) show the instantaneous frequency estimation for 1 bar of pressure. As we can see in Figure 7(a), there is an obvious spike appear at the time of 0.3701s that represent the outlet position. Meanwhile, in Figure 6(b), two spikes appear at the time of $0.1793 \mathrm{~s}$ and $0.2692 \mathrm{~s}$ that can be concluded as leak and outlet position respectively.

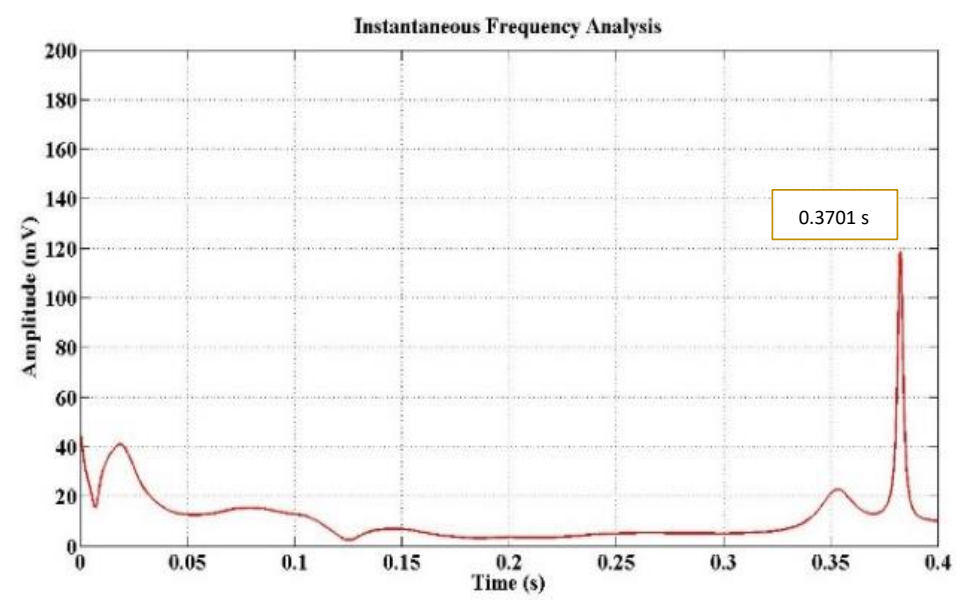

(a) 


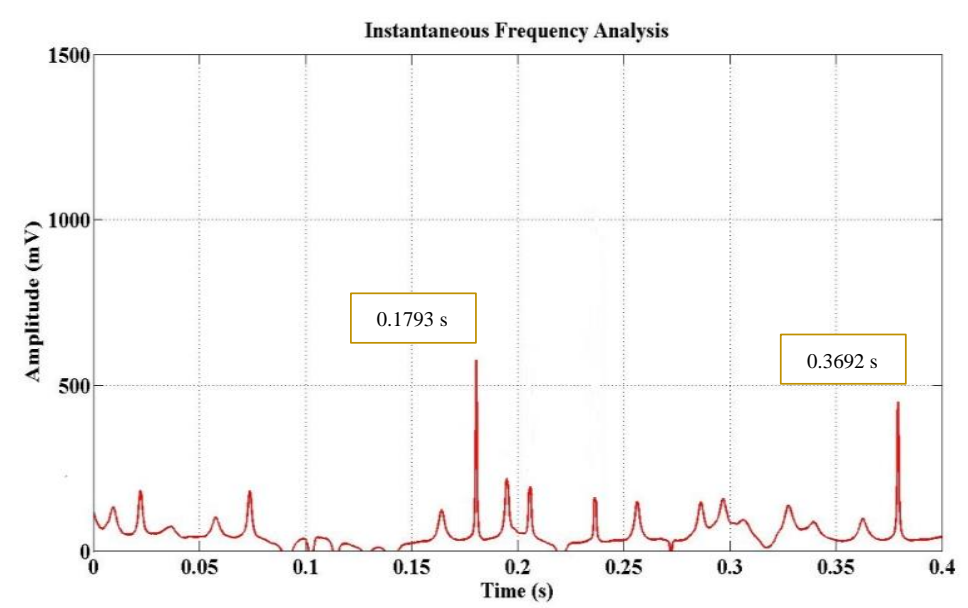

(b)

Fig. 7. (a) IF for 1 bar no leak data, (b) IF for 1 bar leak data

\subsection{Instantaneous Frequency Estimation of 3 bar Signal}

Figure 8(a) and Figure $8(\mathrm{~b})$ show the instantaneous frequency analysis using HT for 3 bar water pressure. For Figure $8(a)$, there is a spike that occurs at the time of 0.3489 s nearly to the outlet position. Meanwhile, in Figure $8(\mathrm{~b})$ the two peaks appear at $0.1887 \mathrm{~s}$ and $0.3618 \mathrm{~s}$ respectively. This can assume as the position of leak and outlet respectively. From the observation, we can see the there were peak appear at the early period around $0.001 \mathrm{~s}$ for both leak and no leak signal. This is because, at the high pressure, the solenoid valve generate high pressure wof water hammer inside the pipeline. Thus, this will be seen clearly after the instantaneous frequency analysis.

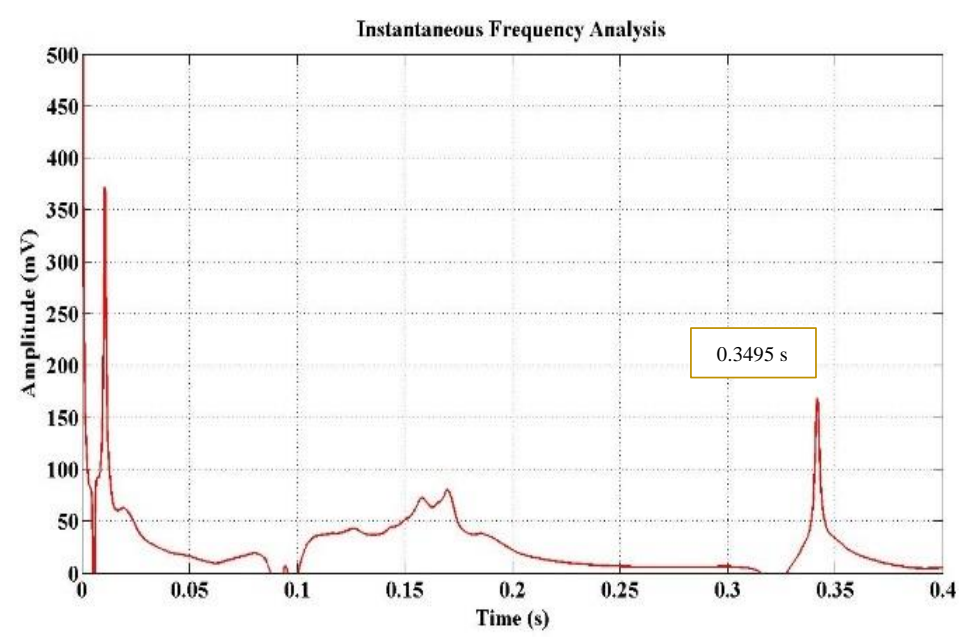

(a) 


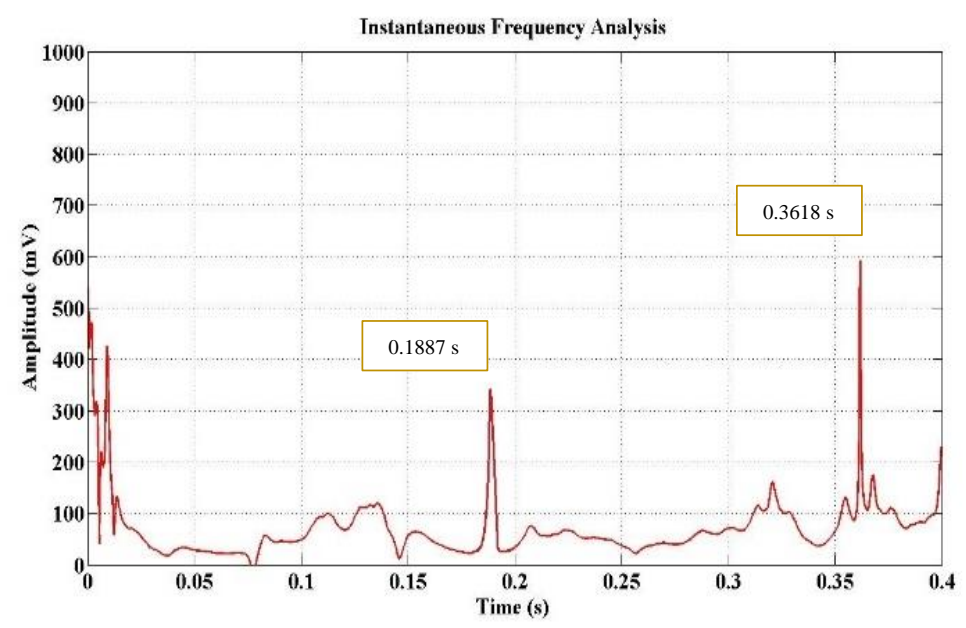

(b)

Fig. 8. (a) IF for 3 bar no leak data. (b) IF for 3 bar leak data

Table 1 summarize all the result of the features extracted from the pipeline network for two different level of pressure and two features. As we can see that all the features extracted successfully from the raw data captured using the pressure sensor. After undergoes decomposition using CEMMD and instantaneous frequency analysis using HT, all the spikes can be seen clearly in the signal. As referenced in Table 1, all features show uniform low-value percentages of error that were only below $4 \%$. The value of percentage of error can be said as acceptable with just only under $4 \%$ for all features located along the pipeline. Thus, the accuracy of the position of all features shows that the method of CEEMD and HT very reliable to be used in leak detection applications either on a lab-scale or in a real water distribution system.

Table 1

Percentage of error for all features

\begin{tabular}{|c|c|c|c|c|c|c|c|c|c|}
\hline \multirow[t]{2}{*}{ Pressure } & \multirow[t]{2}{*}{ Type } & \multicolumn{2}{|c|}{$\begin{array}{l}\text { Time analyzed } \\
\text { (s) }\end{array}$} & \multicolumn{2}{|c|}{$\begin{array}{l}\text { Distance analyzed } \\
\text { (m) }\end{array}$} & \multicolumn{2}{|c|}{$\begin{array}{l}\text { Distance measured } \\
\text { (m) }\end{array}$} & \multicolumn{2}{|c|}{$\begin{array}{l}\text { Percentage error } \\
\text { (\%) }\end{array}$} \\
\hline & & Leak & Outlet & Leak & Outlet & Leak & Outlet & Leak & Outlet \\
\hline \multirow[t]{2}{*}{1 bar } & No leak & - & 0.3701 & - & 96.95 & - & 93.40 & - & 3.80 \\
\hline & Leak & 0.1793 & 0.3692 & 46.97 & 96.78 & 47.87 & & 1.88 & 3.61 \\
\hline \multirow[t]{2}{*}{3 bar } & No leak & - & 0.3495 & - & 91.56 & - & & - & 1.97 \\
\hline & Leak & 0.1887 & 0.3618 & 49.44 & 94.79 & 47.87 & & 3.27 & 1.48 \\
\hline
\end{tabular}

\section{Conclusions}

As a conclusion, The successful of this method can be seen from the result presented in this article. The result show the highest error was only 3.80\% from the no leak and 1 bar signal. Thus, it is proved that the HHT method with the combination of CEEMD and HT show great success as a method to remove noise and unwanted signal that contaminated the true signal. With only error less than $4 \%$ for any features detected, this leak detection method was realibale to be used in the reallife application to locate pipeline failure for above and underground pipeline system. In the future, it is possible to use this method of signal processing on a field test of real water distribution pipeline system. 


\section{Acknowledgment}

This research work is completely financed by Malaysia Pahang University by the provision of funds and facilities. The author also acknowledges the support by the University Malaysia Pahang for providing the internal grant (RDU190346) and also the Ministry of Higher Education (MOHE), for providing grant FRGS/1/2017/TK03/UMP/02/1(RDU170121).

\section{References}

[1] Stafford, M., N. Williams, and Health and Safety Executive, London (United Kingdom). "Pipeline leak detection study." HSE Books London (1996).

[2] Zaki, Muhammad Mirza Mohd, Turki Al Qahtani, Noorfaizal Yidris, Shamsuddin Sulaiman, Ahmad Hamdan Ariffin, Mohd Saffuan Yaakob, and Kamarul Arifin Ahmad. "Design and Analysis of a Water Pipe Leakage Sensor." CFD Letters 12, no. 9 (2020): 51-59. https://doi.org/10.37934/cfdl.12.9.5159

[3] PiRemli, M. A., M. F. Ghazali, W. H. Azmi, M. F. M. Yusof, Hanafi M. Yusop, and M. L. Azmi. "Implementation of Ikaz with Teager-Kaiser energy operator in solving leakage problem using synthetic signal." In IOP Conference Series: Materials Science and Engineering, vol. 469, no. 1, p. 012094. IOP Publishing, 2019. https://doi.org/10.1088/1757899X/469/1/012094

[4] Whaley, R. S., R. E. Nicholas, and J. D. Van Reet. "Tutorial on software based leak detection techniques." PSIG Pipeline Simulation Interest Group (1992).

[5] Abd Gani, Ashraf, and Roszilah Hamid. "Undergrounds Water Pipe Mapping using Ground Penetrating Radar and Global Positioning System." Journal of Advanced Research in Applied Mechanics 50, no. 1 (2018): 1-11.

[6] Turner, N. C. "Hardware and software techniques for pipeline integrity and leak detection monitoring." In Offshore Europe. Society of Petroleum Engineers, 1991. https://doi.org/10.2118/23044-MS

[7] Hough, J. E. "Leak testing of pipelines uses pressure and acoustic velocity." Oil \& Gas Journal 86, no. 47 (1988): 35 41.

[8] Klein, W. R. "Acoustic leak detection." American Society of Mechanical Engineers, Petroleum Division 55 (1993): $57-$ 61.

[9] Colombo, Andrew F., Pedro Lee, and Bryan W. Karney. "A selective literature review of transient-based leak detection methods." Journal of Hydro-Environment Research 2, no. 4 (2009): 212-227. https://doi.org/10.1016/i.jher.2009.02.003

[10] Mpesha, Witness, Sarah L. Gassman, and M. Hanif Chaudhry. "Leak detection in pipes by frequency response method." Journal of Hydraulic Engineering 127, no. 2 (2001): 134-147. https://doi.org/10.1061/(ASCE)07339429(2001)127:2(134)

[11] Duan, Huan-Feng, Pedro J. Lee, Mohamed S. Ghidaoui, and Yeou-Koung Tung. "Essential system response information for transient-based leak detection methods." Journal of Hydraulic Research 48, no. 5 (2010): 650-657. https://doi.org/10.1080/00221686.2010.507014

[12] Duan, Huan-Feng, Pedro J. Lee, Mohamed S. Ghidaoui, and Yeou-Koung Tung. "Leak detection in complex series pipelines by using the system frequency response method." Journal of Hydraulic Research 49, no. 2 (2011): 213 221. https://doi.org/10.1080/00221686.2011.553486

[13] Meniconi, Silvia, Bruno Brunone, Marco Ferrante, and Christian Massari. "Potential of transient tests to diagnose real supply pipe systems: What can be done with a single extemporary test." Journal of Water Resources Planning and Management 137, no. 2 (2011): 238-241. https://doi.org/10.1061/(ASCE)WR.1943-5452.0000098

[14] Wang, Xun, Mohamed S. Ghidaoui, and Pedro J. Lee. "Linear model and regularization for transient wave-based pipeline-condition assessment." Journal of Water Resources Planning and Management 146, no. 5 (2020): 04020028. https://doi.org/10.1061/(ASCE)WR.1943-5452.0001205

[15] Yusop, Hanafi M., M. F. Ghazali, M. F. M Yusof, and W. S. W Hamat. "Improvement of Cepstrum Analysis for the Purpose to Detect Leak, Feature and Its Location in Water Distribution System based on Pressure Transient Analysis." Journal of Mechanical Engineering 4, no. 4 (2017): 103-122.

[16] Torres, María E., Marcelo A. Colominas, Gastón Schlotthauer, and Patrick Flandrin. "A complete ensemble empirical mode decomposition with adaptive noise." In 2011 IEEE International Conference on Acoustics, Speech and Signal Processing (ICASSP), pp. 4144-4147. IEEE, 2011. https://doi.org/10.1109/ICASSP.2011.5947265

[17] Huang, Norden E., Zheng Shen, Steven R. Long, Manli C. Wu, Hsing H. Shih, Quanan Zheng, Nai-Chyuan Yen, Chi Chao Tung, and Henry H. Liu. "The empirical mode decomposition and the Hilbert spectrum for nonlinear and nonstationary time series analysis." Proceedings of the Royal Society of London. Series A: Mathematical, Physical and Engineering Sciences 454, no. 1971 (1998): 903-995. https://doi.org/10.1098/rspa.1998.0193 
[18] Luukko, Perttu JJ, Jouni Helske, and Esa Räsänen. "Introducing libeemd: A program package for performing the ensemble empirical mode decomposition." Computational Statistics 31, no. 2 (2016): 545-557. https://doi.org/10.1007/s00180-015-0603-9

[19] Huang, Norden E., and Zhaohua Wu. "A review on Hilbert-Huang transform: Method and its applications to geophysical studies." Reviews of Geophysics 46, no. 2 (2008). https://doi.org/10.1029/2007RG000228

[20] Yeh, Jia-Rong, Jiann-Shing Shieh, and Norden E. Huang. "Complementary ensemble empirical mode decomposition: A novel noise enhanced data analysis method." Advances in Adaptive Data Analysis 2, no. 02 (2010): $135-156$. https://doi.org/10.1142/S1793536910000422

[21] Lu, Jia, Xing Chen, and Shuo Feng. "A GPS Time Series Prediction Model Based on CEEMD." Journal of Advances in Computer Networks 4, no. 1 (2016). https://doi.org/10.18178/JACN.2016.4.1.206

[22] Lu, Yanfei, Rui Xie, and Steven Y. Liang. "CEEMD-assisted bearing degradation assessment using tight clustering." The International Journal of Advanced Manufacturing Technology 104, no. 1 (2019): 1259-1267. https://doi.org/10.1007/s00170-019-04078-2

[23] Liu, Fuzheng, Junwei Gao, and Huabo Liu. "The feature extraction and diagnosis of rolling bearing based on CEEMD and LDWPSO-PNN." IEEE Access 8 (2020): 19810-19819. https://doi.org/10.1109/ACCESS.2020.2968843

[24] Ghazali, Mohd Fairusham. "Leak detection using instantaneous frequency analysis." PhD diss., University of Sheffield, 2012.

[25] Yusop, Hanafi M., M. F. Ghazali, M. F. M. Yusof, MA Pi Remli, and M. H. Kamarulzaman. "Pipe leak diagnostic using high frequency piezoelectric pressure sensor and automatic selection of intrinsic mode function." In IOP Conference Series: Materials Science and Engineering, vol. 257, no. 1, p. 012091. IOP Publishing, 2017. https://doi.org/10.1088/1757-899X/257/1/012091

[26] PiRemli, M. A., M. F. Ghazali, W. H. Azmi, M. F. M. Yusof, Hanafi M. Yusof, and M. L. Azmi. "Leak localization using empirical mode decomposition and Teiger-Kaiser energy operator analysis based on pressure transient signal: experimental study." In IOP Conference Series: Materials Science and Engineering, vol. 469, no. 1, p. 012093. IOP Publishing, 2019. https://doi.org/10.1088/1757-899X/469/1/012093

[27] Ackroyd, M. H. "Instantaneous and time-varying spectraHan introduction." Radio and Electronic Engineer 39, no. 3 (1970): 145-152. https://doi.org/10.1049/ree.1970.0022

[28] Griffiths, L. "Rapid measurement of digital instantaneous frequency." IEEE Transactions on Acoustics, Speech, and Signal Processing 23, no. 2 (1975): 207-222. https://doi.org/10.1109/TASSP.1975.1162662

[29] Gupta, Madhu Sudan. "Definition of instantaneous frequency and frequency measurability." American Journal of Physics 43, no. 12 (1975): 1087-1088. https://doi.org/10.1119/1.9931

[30] Jones, G., and B. Boashash. "On the concepts of instantaneous frequency time-delay instantaneous bandwidth and their relation to time-frequency distributions." In Proceedings of IEEE Transactions on Acoustics, Speech, and Signal Processing 90, pp. 2467-2470. 1990.

[31] Rihaczek, A. "Signal energy distribution in time and frequency." IEEE Transactions on Information Theory 14, no. 3 (1968): 369-374. https://doi.org/10.1109/TIT.1968.1054157

[32] Ghazali, M. F., S. B. M. Beck, J. D. Shucksmith, J. B. Boxall, and W. J. Staszewski. "Comparative study of instantaneous frequency based methods for leak detection in pipeline networks." Mechanical Systems and Signal Processing 29 (2012): 187-200. https://doi.org/10.1016/i.ymssp.2011.10.011 